Virginia Commonwealth University vCU Scholars Compass

2011

\title{
Hybrid spintronics and straintronics: A magnetic technology for ultra low energy computing and signal processing
}

\author{
Kuntal Roy \\ Virginia Commonwealth University, royk@vcu.edu \\ Supriyo Bandyopadhyay \\ VirginiaCommonwealth University, sbandy@vcu.edu \\ Jayasimha Atulasimha \\ Virginia Commonwealth University, jatulasimha@vcu.edu
}

Follow this and additional works at: http://scholarscompass.vcu.edu/egre_pubs

Part of the Electrical and Computer Engineering Commons

Roy, K., Bandyopadhyay, S., Atulasimha, J. Hybrid spintronics and straintronics: A magnetic technology for ultra low energy computing and signal processing. Applied Physics Letters, 99, 063108 (2011). Copyright (C) 2011 AIP Publishing LLC.

\section{Downloaded from}

http://scholarscompass.vcu.edu/egre_pubs/46 


\title{
Hybrid spintronics and straintronics: A magnetic technology for ultra low energy computing and signal processing
}

\author{
Kuntal Roy, ${ }^{1, a)}$ Supriyo Bandyopadhyay, ${ }^{1}$ and Jayasimha Atulasimha ${ }^{2}$ \\ ${ }^{1}$ Department of Electrical and Computer Engineering, Virginia Commonwealth University, Richmond, \\ Virginia 23284, USA \\ ${ }^{2}$ Department of Mechanical and Nuclear Engineering, Virginia Commonwealth University, Richmond, \\ Virginia 23284, USA
}

(Received 11 January 2011; accepted 25 July 2011; published online 9 August 2011)

\begin{abstract}
The authors show that the magnetization of a 2-phase magnetostrictive/piezoelectric multiferroic single-domain shape-anisotropic nanomagnet can be switched with very small voltages that generate strain in the magnetostrictive layer. This can be the basis of ultralow power computing and signal processing. With appropriate material choice, the energy dissipated per switching event can be reduced to $\sim 45 \mathrm{kT}$ at room temperature for a switching delay of $\sim 100 \mathrm{~ns}$ and $\sim 70 \mathrm{kT}$ for a switching delay of $\sim 10 \mathrm{~ns}$, if the energy barrier separating the two stable magnetization directions is $\sim 32 \mathrm{kT}$. Such devices can be powered by harvesting energy exclusively from the environment without the need for a battery. (C) 2011 American Institute of Physics. [doi:10.1063/1.3624900]
\end{abstract}

The primary obstacle to continued downscaling of digital electronic devices in accordance with Moore's law is the excessive energy dissipation that takes place in the device during switching of bits. Every charge-based device [e.g., metal-oxide-semiconductor field-effect-transistor (MOSFET)] has a fundamental shortcoming in this regard. They are switched by injecting or extracting an amount of charge $\Delta Q$ from the device's active region with a potential gradient $\Delta V$, leading to an inevitable energy dissipation of $\Delta Q \times \Delta V$. Spin based devices, on the other hand, are switched by flipping spins without moving any charge in space $(\Delta Q=0)$ and causing a current flow. Although some energy is still dissipated in flipping spins, it can be considerably less than the energy $\Delta Q \times \Delta V$ associated with current flow. This gives "spin" an advantage over "charge" as a state variable.

Recently, it has been shown that the minimum energy dissipated to switch a charge-based device like a transistor at a temperature $T$ is $\sim N k T \ln (1 / p)$, where $N$ is the number of information carriers (electrons or holes) in the device and $p$ is the bit error probability. ${ }^{1}$ On the other hand, the minimum energy dissipated to switch a single-domain nanomagnet (which is a collection of $M$ spins) can be only $\sim k T \ln (1 / p)$, since the exchange interaction between spins makes $M$ spins rotate together in unison like a giant classical spin. ${ }^{1,2}$ This gives the magnet an advantage over the transistor.

Unfortunately, the magnet's advantage is lost if the method adopted to switch, it is so inefficient that the energy dissipated in the switching circuit far exceeds the energy dissipated in the magnet. Regrettably, this is often the case. A magnet is usually flipped with either a magnetic field generated by a current ${ }^{3}$ or a spin polarized current exerting either a spin transfer torque ${ }^{4}$ or causing domain-wall motion. ${ }^{5}$ The energy dissipated to switch a magnet with current-generated magnetic field was reported in Ref. 3 as $10^{11}-10^{12} \mathrm{kT}$ for a switching delay of $\sim 1 \mu \mathrm{s}$, which clearly makes it impractical. In fact, it will make the magnet inferior to the transistor

${ }^{a)}$ Electronic mail: royk@vcu.edu. which can be switched in sub-ns while dissipating $10^{7}-10^{8}$ kT of energy in a circuit. ${ }^{6}$ Domain-wall motion induced by a spin-polarized current can switch a nanomagnet in 2 ns while dissipating $10^{4}-10^{5} \mathrm{kT}$ of energy, ${ }^{7}$ but there is still a need to identify more energy-efficient mechanisms for switching a magnet.

Recently, we have shown that the magnetization of a shape-anisotropic piezoelectric/magnetostrictive multiferroic nanomagnet can be switched with a small voltage applied to the piezoelectric layer. ${ }^{8}$ Such multiferroic systems have now become commonplace ${ }^{9-11}$ and there are proposals for using them in magnetic logic and memory. ${ }^{8,12}$ In this method, the electrostatic potential generates uniaxial strain in the piezoelectric layer, and that is elastically transferred to the magnetostrictive layer if the latter is considerably thinner. The nanomagnet is clamped along the hard axis. This makes the magnetization of the magnetostrictive layer rotate. Such rotations have been demonstrated experimentally. ${ }^{10}$

Consider an ellipsoidal multiferroic magnet with uniaxial shape anisotropy as shown in Fig. 1. The piezoelectric layer is $40 \mathrm{~nm}$ thick, and the magnetostrictive layer is $10 \mathrm{~nm}$ thick, which is thin enough that strain does not relax. We assume that the piezoelectric layer is lead-zirconate-titanate (PZT) and the magnetostrictive layer is polycrystalline nickel or cobalt or Terfenol-D. For Terfenol-D, the major axis is assumed to be $\sim 102 \mathrm{~nm}$ and the minor axis is $\sim 98 \mathrm{~nm}$. Because of shape anisotropy, the two magnetization orientations parallel to the easy axis (major axis of the ellipse or the z-axis) are stable and can store the binary bits 0 and 1 . We keep the potential energy barrier between these two orientations (i.e., the shape anisotropy barrier) $0.8 \mathrm{eV}$ or $\sim 32 \mathrm{kT}$ at room temperature by choosing the appropriate parameters, which makes the static bit error probability $e^{-32}$.

Let us assume that the magnetization is initially oriented along the $-\mathrm{z}$-axis. Our task is to switch the nanomagnet so that the final orientation is along the $+\mathrm{z}$-axis. We do this by applying a voltage $V$ across the thickness of the piezoelectric layer that generates uniaxial stress along the easy axis 

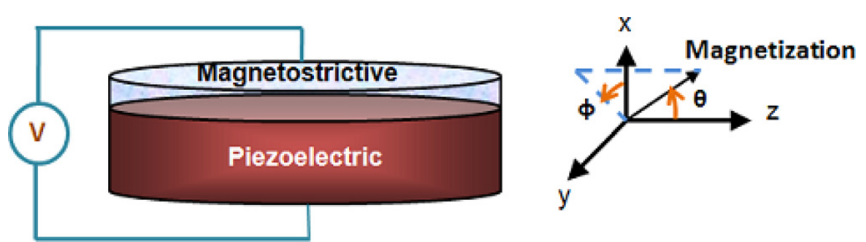

FIG. 1. (Color online) An elliptical multiferroic nanomagnet stressed with an applied voltage.

(z-axis) via $d_{31}$ coupling. The energy dissipated in the switching circuit during turn-on is $(1 / 2) C V^{2}$ while that dissipated during turn-off is $(1 / 2) C V^{2}$, where $C$ is the capacitance of the piezoelectric layer plus any line capacitance. Since the piezoelectric layer has a very large relative dielectric constant (1000), its capacitance will dominate over the line capacitance which can be neglected.

There is an additional dissipation $E_{d}$ in the nanomagnet due to Gilbert damping. ${ }^{13}$ The total energy dissipated in the switching process is, therefore, $E_{\text {total }}=C V^{2}+E_{d}$. Thus, in order to calculate $E_{\text {total }}$ as a function of switching delay, we have to calculate four quantities: (1) the stress needed to switch the magnetization within a given delay, (2) the voltage $V$ needed to generate this stress, (3) the capacitance $C$, and (4) $E_{d}$ which is calculated by following the prescription of Ref. 13 .

In order to find the stress $\sigma$ required to switch a magnetostrictive nanomagnet in a given time delay $\tau$, we solve the Landau-Lifshitz-Gilbert (LLG) equation for a single-domain magnetostrictive nanomagnet subjected to stress $\sigma$. We then relate $\sigma$ to the strain $\varepsilon$ in the nanomagnet from Hooke's law ( $\varepsilon=\sigma / Y$, where $Y$ is the Young's modulus of the nanomagnet) and find the voltage $V$ that generates that strain in the piezoelectric layer based on its $d_{31}$ coefficient and thickness. Finally, we calculate the capacitance of the multiferroic system by treating it as a parallel-plate capacitor. This allows us to find the energy dissipated in the switching circuit $\left(\mathrm{CV}^{2}\right)$ as a function of the switching delay $\tau$.

In the supplementary material accompanying this letter, ${ }^{14}$ we show that both stress and shape anisotropy act like a torque on the magnetization of the nanomagnet. This torque per unit volume of the nanomagnet is

$$
\mathbf{T}_{\mathbf{E}}(t)=-\mathbf{n}_{\mathbf{m}}(t) \times \nabla E[\theta(t), \phi(t)],
$$

where $E[\theta(t), \phi(t)]$ is the total potential energy of the nanomagnet at an instant of time $t$. It is the sum of shape anisotropy energy and stress anisotropy energy, both of which depend on the magnetization orientation at the given instant determined by the polar angle $\theta(t)$ and azimuthal angle $\phi(t)$ of the magnetization vector which is assumed to be in the radial direction.

We can write the torque as

$$
\begin{aligned}
\mathbf{T}_{\mathbf{E}}(t)= & -\{2 B(\phi(t)) \sin \theta(t) \cos \theta(t)\} \hat{\mathbf{e}}_{\phi} \\
& -\left\{B_{0 e}(\phi(t)) \sin \theta(t)\right\} \hat{\mathbf{e}}_{\theta},
\end{aligned}
$$

where $\hat{\mathbf{e}}_{\theta}$ and $\hat{\mathbf{e}}_{\phi}$ are unit vectors in the $\theta$ - and $\phi$-directions, and

$$
B_{0}(\phi(t))=\frac{\mu_{0}}{2} M_{s}^{2} \Omega\left[N_{x x} \cos ^{2} \phi(t)+N_{y y} \sin ^{2} \phi(t)-N_{z z}\right],
$$

$$
\begin{gathered}
B_{\text {stress }}=(3 / 2) \lambda_{s} \sigma \Omega, \\
B(\phi(t))=B_{0}(\phi(t))+B_{\text {stress }}, \\
B_{0 e}(\phi(t))=\frac{\mu_{0}}{2} M_{s}^{2} \Omega\left(N_{x x}-N_{y y}\right) \sin (2 \phi(t)) .
\end{gathered}
$$

Here $M_{s}$ is the saturation magnetization of the nanomagnet, $\Omega$ is its volume, $\mu_{0}$ is the permeability of free space, $\lambda_{s}$ is the magnetostrictive coefficient of the magnetostrictive layer, and $N_{\beta \beta}$ is the demagnetization factor in the $\beta$ direction, which can be calculated from the shape and size of the nanomagnet (see the supplementary material ${ }^{14}$ ).

The magnetization dynamics of the single-domain nanomagnet (neglecting thermal fluctuations) is described by the LLG equation

$$
\frac{d \mathbf{n}_{\mathbf{m}}(t)}{d t}+\alpha\left(\mathbf{n}_{\mathbf{m}}(t) \times \frac{d \mathbf{n}_{\mathbf{m}}(t)}{d t}\right)=\frac{\gamma}{M_{V}} \mathbf{T}_{\mathbf{E}}(t),
$$

where $\mathbf{n}_{\mathbf{m}}(t)$ is the normalized magnetization, $\alpha$ is the dimensionless phenomenological Gilbert damping constant, $\gamma=2 \mu_{B} \mu_{0} / \mathrm{h}$ is the gyromagnetic ratio for electrons, and $M_{V}=\mu_{0} M_{s} \Omega$.

From this equation, we can derive two coupled equations that describe the $\theta$ - and $\phi$-dynamics,

$$
\begin{gathered}
\left(1+\alpha^{2}\right) \theta^{\prime}(t)=-\frac{\gamma}{M_{V}}\left[B_{0 e}(\phi(t)) \sin \theta(t)\right. \\
+2 \alpha B(\phi(t)) \sin \theta(t) \cos \theta(t)], \\
\left(1+\alpha^{2}\right) \phi^{\prime}(t)=\frac{\gamma}{M_{V}}\left[\alpha B_{0 e}(\phi(t))-2 B(\phi(t)) \cos \theta(t)\right] \\
(\sin \theta(t) \neq 0) .
\end{gathered}
$$

Clearly, the $\theta$ - and $\phi$-motions are coupled and hence these equations have to be solved numerically. We assume that the initial orientation of the nanomagnet is close to the $-\mathrm{z}$-axis $\left(\theta=179^{\circ}\right)$. It cannot be exactly along the $-\mathrm{z}$-axis $\left(\theta=180^{\circ}\right)$ since then the torque acting on it will be zero [see Eq. (2)] and the magnetization will never rotate under any stress. Similarly, we cannot make the final state align exactly along the $+\mathrm{z}$-axis $\left(\theta=0^{\circ}\right)$ in a reasonable time since there too the torque vanishes. Hence, we assume that the final state is $\theta=1^{\circ}$. Thus, both initial and final states are $1^{\circ}$ off from the easy axis. Thermal fluctuations can easily deflect the magnetization by $1^{\circ}$ (see Ref. 15).

We apply the voltage generating stress abruptly at time $t=0$. This rotates the magnetization away from near the easy axis $\left(\theta=179^{\circ}\right)$ to the new energy minimum at $\theta=90^{\circ}$. We maintain the stress until $\theta$ reaches $90^{\circ}$ which places the magnetization approximately along the in-plane hard axis (y-axis). Then, we reduce the voltage to zero abruptly. Subsequently, shape anisotropy takes over and the magnetization vector rotates towards the easy axis since that now becomes the minimum energy state. The question is which direction along the easy axis will the magnetization vector relax to. Is it the $-\mathrm{z}$-axis at $\theta=179^{\circ}$ (wrong state) or the $+\mathrm{z}$-axis at $\theta=1^{\circ}$ (correct state)? That is determined by the sign of $B_{0 e}(\phi(t))$ when $\theta$ reaches $90^{\circ}$. If $\phi$ at that instant is less than 


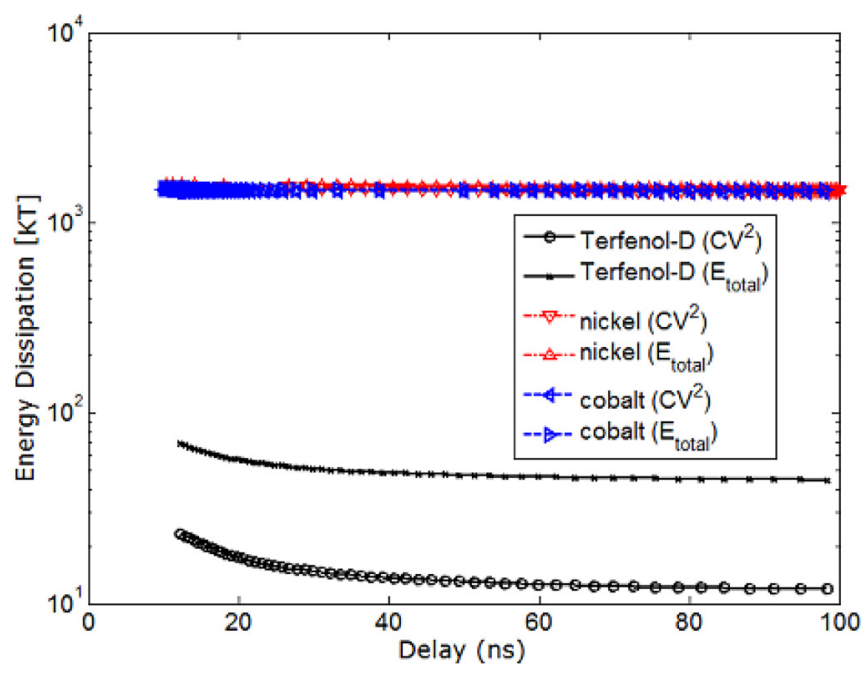

FIG. 2. (Color online) Energy dissipated in the switching circuit $\left(C V^{2}\right)$ and the total energy dissipated $\left(E_{\text {total }}\right)$ as functions of delay for three different materials used as the magnetostrictive layer in the multiferroic nanomagnet.

$90^{\circ}$, then $B_{0 e}(\phi(t))$ is positive which makes the time derivative of $\theta$ negative (see Eq. (5)), so that $\theta$ continues to decrease and the magnetization reaches the correct state close to the $+\mathrm{z}$-axis. The coupled $\theta$ - and $\phi$-dynamics ensures that this is the case as long as the stress exceeds a minimum value. Thus, successful switching requires a minimum stress.

Once we have found the switching delay $\tau$ for a given stress $\sigma$ by solving Eqs. (5) and (6), we can invert the relationship to find $\sigma$ versus $\tau$ and hence the energy dissipated versus $\tau$. This is shown in Fig. 2 where we plot the energy dissipated in the switching circuit $\left(C V^{2}\right)$, as well as the total energy dissipated $\left(E_{\text {total }}\right)$ versus delay for three different magnetostrictive materials. For Terfenol-D, the stress required to switch in $100 \mathrm{~ns}$ is $1.92 \mathrm{MPa}$ and that required to switch in $10 \mathrm{~ns}$ is $2.7 \mathrm{MPa}$.

Note that for a stress of $1.92 \mathrm{MPa}$, the stress anisotropy energy $B_{\text {stress }}$ is $32.7 \mathrm{kT}$ while for $2.7 \mathrm{MPa}$, it is $46.2 \mathrm{kT}$. As expected, they are larger than the shape anisotropy barrier of $\sim 32 \mathrm{kT}$ which had to be overcome by stress to switch. A larger excess energy is needed to switch faster. The energy dissipated and lost as heat in the switching circuit $\left(C V^{2}\right)$ is only $12 \mathrm{kT}$ for a delay of $100 \mathrm{~ns}$ and $23.7 \mathrm{kT}$ for a delay of $10 \mathrm{~ns}$. The total energy dissipated is $45 \mathrm{kT}$ for a delay of 100 ns and $70 \mathrm{kT}$ for a delay of $10 \mathrm{~ns}$. Note that in order to increase the switching speed by a factor of 10 , the dissipation needs to increase by a factor of 1.6. Therefore, dissipation increases sub-linearly with speed, which bodes well for energy efficiency.

With a nanomagnet density of $10^{10} \mathrm{~cm}^{-2}$ in a memory or logic chip, the dissipated power density would have been only $2 \mathrm{~mW} / \mathrm{cm}^{2}$ to switch in $100 \mathrm{~ns}$ and $30 \mathrm{~mW} / \mathrm{cm}^{2}$ to switch in $10 \mathrm{~ns}$, if $10 \%$ of the magnets switch at any given time (10\% activity level). Note that unlike transistors, magnets have no leakage and no standby power dissipation, which is an important additional benefit.

Such extremely low power and yet high density magnetic logic and memory systems, composed of multiferroic nanomagnets, can be powered by existing energy harvesting systems ${ }^{16-19}$ that harvest energy from the environment without the need for an external battery. These processors are uniquely suitable for implantable medical devices, e.g., those implanted in a patient's brain that monitor brain signals to warn of impending epileptic seizures. They can run on energy harvested from the patient's body motion. For such applications, 10-100 ns switching delay is adequate. These hybrid spintronic/straintronic processors can be also incorporated in "wrist-watch" computers powered by arm movement, buoy-mounted computers for tsunami monitoring (or naval applications) that harvest energy from sea waves, or structural health monitoring systems for bridges and buildings that are powered solely by mechanical vibrations due to wind or passing traffic.

${ }^{1}$ S. Salahuddin and S. Datta, Appl. Phys. Lett. 90, 093503 (2007).

${ }^{2}$ R. P. Cowburn, D. K. Koltsov, A. O. Adeyeye, M. E. Welland, and D. M. Tricker, Phys. Rev. Lett. 83, 1042 (1999).

${ }^{3}$ M. T. Alam, M. J. Siddiq, G. H. Bernstein, M. T. Niemier, W. Porod, and X. S. Hu, IEEE Trans. Nanotechnol. 9, 348 (2010).

${ }^{4}$ D. C. Ralph and M. D. Stiles, J. Magn. Magn. Mater. 320, 1190 (2008).

${ }^{5}$ M. Yamanouchi, D. Chiba, F. Matsukura, and H. Ohno, Nature (London) 428, 539 (2004).

${ }^{6}$ See CORE9GPLL_HCMOS9_TEC_4.0 Databook for information about propagation delay and energy dissipation, UNICAD2.4, STMicroelectronics (2003).

${ }^{7}$ S. Fukami, T. Suzuki, K. Nagahara, N. Ohshima, Y. Ozaki, S. Saito, R. Nebashi, N. Sakimura, H. Honjo, K. Mori, C. Igarashi, S. Miura, N. Ishiwata, and T. Sugibayashi, Dig. Tech. Pap. - Symp. VLSI Technol. 2009, 230.

${ }^{8}$ J. Atulasimha and S. Bandyopadhyay, Appl. Phys. Lett. 97, 173105 (2010).

${ }^{9}$ F. Zavaliche, T. Zhao, H. Zheng, F. Straub, M. P. Cruz, P. L. Yang, D. Hao, and R. Ramesh, Nano Lett. 7, 1586 (2007).

${ }^{10}$ T. Brintlinger, S. H. Lim, K. H. Baloch, P. Alexander, Y. Qi, J. Barry, J. Melngailis, L. Salamanca-Riba, I. Takeuchi, and J. Cumings, Nano Lett. 10, 1219 (2010).

${ }^{11}$ W. Eerenstein, N. D. Mathur, and J. F. Scott, Nature (London) 442, 759 (2006).

${ }^{12}$ S. A. Wolf, J. Lu, M. R. Stan, E. Chen, and D. M. Treger, Proc. IEEE 98 , 2155 (2010).

${ }^{13}$ B. Behin-Aein, S. Salahuddin, and S. Datta, IEEE Trans. Nanotechnol. 8, 505 (2009).

${ }^{14}$ See supplementary material at http://dx.doi.org/10.1063/1.3624900 for detailed derivations and additional simulation results.

${ }^{15}$ D. E. Nikonov, G. I. Bourianoff, G. Rowlands, and I. N. Krivorotov, J. Appl. Phys. 107, 113910 (2010).

${ }^{16}$ S. Roundy, Ph.D. thesis, Mech. Eng., University of California, Berkeley, California, 2003.

${ }^{17}$ S. R. Anton and H. A. Sodano, Smart Mater. Struct. 16, R1 (2007).

${ }^{18}$ F. Lu, H. P. Lee, and S. P. Lim, Smart Mater. Struct. 13, 57 (2004).

${ }^{19}$ Y. B. Jeon, R. Sood, J. Jeong, and S. G. Kim, Sens. Actuators, A 122, 16 (2005). 